\title{
Variability of Pause Patterns in English Read Speech of Thai EFL Learners
}

\author{
Soisithorn Isarankura, Ph.D. \\ Assistant Professor, Faculty of Arts \\ Dhurakij Pundit University, Bangkok, Thailand
}

\section{Doi:10.5901/jesr.2013.v3n7p346}

\begin{abstract}
Research suggests that pauses are essential in oral communication in that they render intelligibility and contribute to the improvement of speech comprehension. Unfortunately, the teaching of correct pausing has received little attention in EFL classes. As a result, many Thai learners of English tend to use inappropriate phrasing and pausing, which makes their speech sound unnatural or even hinders communication. This study investigates pause patterns in read speech of two English proficiency levels of Thai learners in comparison with those produced by native English speakers. The opening section provides a brief background of the study and terminology related to pauses in spoken language and research objectives. The second section describes the research design. The results of the study presented in the third section reveal that native speakers paused exclusively at sentence ends. Additional pauses were made at major syntactic boundaries. Inter-speaker variability existed at minor syntactic boundaries. Among Thai learners, the lower proficiency group paused more frequently and produced shorter and improper lengths of information chunks than the higher proficiency group, who read in longer, but more syntactically- and semantically-unified, units and therefore paused less. The findings support previous studies that pause is, to a large extent, affected by syntactic structures. Since correct use of pauses can make a marked improvement of intelligibility in speech production, the pedagogical implication offered from the findings is to support the importance of introducing read-aloud tasks in EFL classes.
\end{abstract}

\section{Introduction}

\subsection{Research Background}

Human speech communication contains both sounds and silence (pause). Pauses normally occur either in read or spontaneous speech in all languages. Generally, pauses of various lengths can occur at the end of the statement or between grammatical units within sentences. The functions of a pause may differ in several respects. Pauses are constrained by physiological and cognitive factors. In speech production, pauses are necessary for a speaker to regain his breath and make time available for the cognitive processes of speech planning. With respect to linguistic functions, speakers or readers can use pauses to segment an utterance into smaller stretches. Effective speakers use appropriate pauses to draw the hearer's attention to meaningful chunks of information they would like to convey. Zellner (1994) argued that pauses are essential in rendering communication intelligibility and also contribute a great deal to the improvement of the learner's speech comprehension.

From the experience of the author in teaching English courses to Thai undergraduate students in Thailand, it has been found that the problems of the students' oral production are mainly at the supra-segmental level (or prosody), which also include the use of pause. Many Thai learners tend to use inappropriate pausing. Several empirical studies of pauses produced by non-native English speakers (e.g. Riazantseva, 2001; Bada, 2006; Bada \& Genc, 2008) revealed that their pause patterns were different from those observed among native English speakers. Consequently, this study was conducted to investigate the pause patterns of Thai learners of English and to compare the results with baseline data on native English speakers. Furthermore, this study also aims to highlight the importance of the teaching of read-aloud tasks in EFL classes in order to enhance the learner's ability to recognize relationships between structural parts of a sentence and to read language using appropriate pausing.

\subsection{Pause, Sense Group and Syntactic Structure}

The occurrence of pause is constrained by a number of factors. Numerous studies (e.g. Zvonik, 2004; Krivokapi, 2008) have been conducted in order to identify the factors that may affect the positions of pause. Beside physiological constraints such as articulation rate and respiratory capacity of each speaker, one of the circumstances that may 
motivate speakers to break up a sentence into smaller stretches is the syntactic component. Sentences can normally be divided into smaller units that are grammatically and semantically related with each other. These units are called 'sense groups'. In speech, the clue to identify a sense group is the pause that occurs before and after it. In principle, no pause appears within a sense group; in other words, a sense group is usually not to be interrupted within a group. If sense group boundaries are marked inappropriately by non-native speakers (NNS), the result is difficulty on the part of the listener to follow the thread of speech (Wennerstrom, 1994). Taylor (1981) asserted that faulty division of sense groups is likely to cause uneven, jerky rhythm in speech. Additional research has shown that syntactic structure plays a role in boundary placement in sense groups and pause location (Selkirk, 1984; Truckenbrodt, 1999). Knowledge of constituent grammar or the ability to recognize relationships between structural parts of a sentence could play a part in the ability to read in appropriate sense groups.

\subsection{Read Speech}

Several studies (e.g. Keating \& Shattuck-Hufnagel, 2002; Zvonik, 2004) report that pause patterns vary by speaking tasks and different genres of spoken language. For example, conversational speech, which is spontaneous in nature, differs in many respects from speech elicited under the constraint of reading a text aloud (often termed as read speech). A speaker speaking spontaneously is confronted by a great number of cognitive constraints, many of which concern the planning decisions such as deciding on a topic, selecting ways to present the topic, sorting out appropriate syntactic structures, selecting lexical items, and so on. Such planning problems result in hesitations, restarts and repetitions, which affect drastically on how boundaries and pauses are realized. As a result, many speakers may produce non-fluent speech which contains more frequent pauses than others.

Krivokapi (2008) maintained that factors determining pause patterns in read speech are fewer and can be more easily controlled than those in spontaneous speech. The reader performing a read-aloud task is not confronted by most of the planning decisions that a speaker speaking spontaneously is confronted with. Because a speaker is given the lexical items and the structure of the whole utterance in advance, "the speech mechanism is set up to make use of such information" (Keating \& Shattuck-Hufnagel, 2002, p.131). The read-aloud task, thus, allows for control over the content of the reading text and the predictability of sense groups. Results from many studies (e.g. Grosjean et al, 1979; Zvonik, 2004; Krivokapi, 2008) indicated that pauses in read speech mainly coincide with syntactic structures and tend to occur at major syntactic boundaries. The process of reading aloud from text requires a division of sentences into syntactic units and the assignment of pauses between those units. Given that pauses in read speech are mainly, if not fully, influenced by syntactic structures, inter-speaker variability in pause patterns should be reduced. In other words, correct pausing should be much more well-defined in read speech than in spontaneous speech. This study, therefore, sought to examine pauses in read speech in relation to syntactic structures of sentences.

\subsection{Research Objective}

The objective of this study is two-fold:

1. To investigate the pause patterns in read speech of Thai EFL learners and to compare the results with baseline data on native English speakers;

2. To examine the extent to which the pause patterns of native English speakers and Thai EFL learners at high and low English proficiency levels exhibit relations to syntactic structures of English sentences.

\section{Methodology}

\subsection{Participants}

The participants in the study consisted of 30 Thai EFL learners and 7 native English speakers. The Thai learners were undergraduate students in the English major program at Dhurakij Pundit University, located in Bangkok, Thailand. These students were selected and classified into 2 English proficiency groups, high and low, based on their relative English proficiency. The classification was based on their scores on an in-house test of English proficiency taken by 195 students (80 seniors and 115 freshmen). Following the test, 15 students with the highest scores and 15 students with the lowest scores were selected and placed into 2 groups: NNS-H and NNS-L, respectively. The native English speaker group consisted of 7 English instructors at the university level in Thailand, representing NS controls. All participants in this study 
were not informed of the purpose of the experiment.

\subsection{Instruments}

This study utilized 2 instruments for data collection:

1. An Aesop fable, The North Wind and The Sun, transcribed by Dr. Lucinda Hart-Gonzalez and adapted for the purpose of this study;

2. The PRAAT sound analyzing software, version 5.1 .15 , for acoustic analysis of pause location and duration in individual recordings.

The Aesop fable used for the read-aloud task contained 119 words in total. Prior to the experiment, the text was analyzed by means of Immediate Constituent (IC) Analysis to divide sentences into successive layers and determine boundaries. Then the analyzed text was marked with the double slash $(I /)$ and the single slash $(I)$ at predetermined strong and weak boundaries, respectively. Strong boundaries include inter-sentential boundaries, defined by punctuation such as a full stop, a semicolon and some cases of embedded clauses. Weak boundaries include most intra-sentential boundaries such as those between phrases. It should be noted that boundaries between phrases that contain a small number of words are, in most cases, less likely pause sites. Examples are those at the subject-predicate juncture when the subject is a pronoun or a simple noun phrase.

\subsection{Data Collection}

The data gathering process was conducted in a language laboratory. The participants were given a copy of the text and were instructed to read it silently with no time constraint to familiarize themselves with the vocabulary and structure. The purpose was to keep information load to the minimum. After the familiarization period, the participants started recording their speech read-aloud at normal speaking rate.

\subsection{Data Analysis}

The analysis was conducted in three stages:

1. Each recording was listened to by 3 listeners in order to identify pause locations. The symbol $(/)$ was marked on a printed transcript where a pause was heard.

2. To support the auditory analysis, the PRAAT sound analyzing software was used to automatically detect pause locations and measure the duration of all the pauses. At the locations where inconsistencies existed among the 3 listeners in the analysis in stage 1, the PRAAT program was conducted manually to verify the results. In this study, pauses of 200 milliseconds (ms) were considered the threshold for data analysis.

3. Following stages 1 and 2, statistical analysis was performed, using descriptive statistics, one-way ANOVA and the Scheffe's method.

\section{Results}

\subsection{Number of PDU}

To give an overall picture of the pause patterns, all chunks between pauses (termed as 'pause-defined units' or PDU in Luksaneeyanawin, 1988) were counted per participant and calculated for mean values and standard deviation as shown in Table 1.

Table 1: Number of PDU by group

\begin{tabular}{|l|l|l|l|l|l|l|l|l|l|l|l|l|l|l|l|l|l|}
\hline Participants & 1 & 2 & 3 & 4 & 5 & 6 & 7 & 8 & 9 & 10 & 11 & 12 & 13 & 14 & 15 & $\bar{x}$ & Std. \\
\hline NS ( $\mathrm{n}=7)$ & 14 & 16 & 16 & 23 & 16 & 20 & 16 & & & & & & & & & 17.29 & 3.094 \\
\hline NNS-H (n=15) & 24 & 25 & 24 & 19 & 24 & 21 & 22 & 27 & 22 & 16 & 19 & 32 & 23 & 22 & 27 & 23.13 & 3.852 \\
\hline NNS-L (n=15) & 31 & 34 & 37 & 26 & 19 & 47 & 32 & 37 & 19 & 26 & 38 & 19 & 33 & 42 & 20 & 30.67 & 8.918 \\
\hline
\end{tabular}

From Table 1, it can be observed that the number of PDU varied among the participants. The native speakers divided the 
119-word text into 17.29 PDUs in average, whereas Thai learners in the high group produced 23.13 PDUs in average, and the low group read the text in 30.67 PDUs. This means that lower proficiency level learners paused more frequently than those with higher English proficiency. The statistical testing results showed significant difference in the number of PDU between the NS group and the NNS-L group, as well as between the two groups of Thai learners. The performance between the NS group and the NNS-H group did not differ significantly, as shown in Table 2 below.

Table 2: Comparisons of the Mean Values of PDUs across Groups

\begin{tabular}{llll}
\hline Group & Mean Diff & Std. & Sig. \\
\hline NS vs. NNS-H & 5.85 & 2.915 & .149 \\
\hline NS vS. NNS-L & $13.38^{*}$ & 2.915 & .000 \\
\hline NNS-H vs. NNS-L & $7.53^{*}$ & 2.325 & .010 \\
\hline
\end{tabular}

${ }^{*} \mathrm{p}<.05$

\subsection{Size of PDU}

The size of PDU (or the number of words per pause) was calculated as the proportion of words in total divided by the number of PDU per participant as displayed in Table 1, and then averaged to obtain the mean value for each sample group.

Table 3: Size of PDU by group

\begin{tabular}{|l|l|l|l|l|l|l|l|l|l|l|l|l|l|l|l|l|l|}
\hline Participants & 1 & 2 & 3 & 4 & 5 & 6 & 7 & 8 & 9 & 10 & 11 & 12 & 13 & 14 & 15 & $\bar{x}$ & Std. \\
\hline NS $(\mathrm{n}=7)$ & 8.5 & 7.4 & 7.4 & 5.2 & 7.4 & 5.9 & 7.4 & & & & & & & & & 7.05 & 1.113 \\
\hline NNS-H $(\mathrm{n}=15)$ & 4.9 & 4.8 & 4.9 & 6.3 & 4.9 & 5.7 & 5.4 & 4.4 & 5.4 & 7.4 & 6.3 & 3.7 & 5.2 & 5.4 & 4.4 & 5.28 & 900 \\
\hline NNS-L $(\mathrm{n}=15)$ & 3.8 & 3.5 & 3.2 & 4.6 & 6.3 & 2.5 & 3.7 & 3.2 & 6.3 & 4.6 & 3.1 & 6.3 & 3.6 & 2.8 & 5.9 & 4.23 & 1.337 \\
\hline
\end{tabular}

With respect to the size of PDU, the analysis in Table 3 revealed that participants in the NS group read the text at the average of 7.05 words per PDU, whereas the NNS-H and NNS-L groups read the text at the average of 5.28 and 4.23 words per pause, respectively. This suggests that the high group had the ability to produce bigger or longer chunks of information and their PDU size was closer to that of the NS group than those with the lower proficiency level. However, statistical testing results revealed, as shown in Table 4, that the performance between the high group and the native speakers differed significantly. Significant difference was also observed between the NS and NNS-L group, but not between the 2 groups of Thai learners.

Table 4: Comparisons of the Mean Values of PDU Size across Groups

\begin{tabular}{lllll}
\cline { 2 - 4 } & Group & Mean Diff & Std. & Sig. \\
\cline { 2 - 5 } & NS vS. NNS-H & $1.77^{*}$ & .519 & .007 \\
\cline { 2 - 5 } & NS vs. NNS-L & $2.82^{*}$ & .519 & .000 \\
\cline { 2 - 5 }$*_{p}<.05$ & NNS-H vs. NNS-L & 1.05 & .414 & .054 \\
\cline { 2 - 5 } & & & &
\end{tabular}

\subsection{Number of PDU and Size of PDU at the Sentence Level}

A more in-depth analysis was further conducted to examine the number of PDU and size of PDU at the sentence level. Table 5 below shows the average number of PDU at the sentence level by group and Table 6 displays PDU size in average. The numbers in parentheses in the first column indicate the number of words in each sentence.

Table 5: Number of PDU by Sentence

\begin{tabular}{llll}
\hline \multirow{2}{*}{ Sentence } & $\bar{x}$ Number of PDU & \multirow{2}{*}{ Sig. Scheffe's Method } \\
\cline { 2 - 3 } & NS (1) & NNS-L (3) & \\
& & \\
& & 349
\end{tabular}




\begin{tabular}{llllll}
\hline Title $(6 \mathrm{w})$ & 1.29 & 1.13 & 1.07 & .396 & - \\
\hline $\mathrm{S} 1(26 \mathrm{w})$ & 2.71 & 4.80 & 6.40 & $.001^{*}$ & $(1)<(3)$ \\
\hline $\mathrm{S} 2(22 \mathrm{w})$ & 3.14 & 3.67 & 5.27 & $.006^{*}$ & $(1)<(3),(2)<(3)$ \\
\hline $\mathrm{S} 3(33 \mathrm{w})$ & 5.14 & 6.73 & 9.53 & $.001^{*}$ & $(1)<(3),(2)<(3)$ \\
\hline $\mathrm{S} 4(16 \mathrm{w})$ & 2.29 & 3.67 & 4.27 & $.010^{*}$ & $(1)<(3)$ \\
\hline $\mathrm{S} 5(16 \mathrm{w})$ & 2.71 & 3.13 & 4.13 & $.011^{*}$ & $(1)<(3)$ \\
\hline${ }^{*} \mathrm{p}<.05$ & & & & &
\end{tabular}

Table 6: Size of PDU by Sentence

\begin{tabular}{|c|c|c|c|c|c|}
\hline \multirow{2}{*}{ Sentence } & \multicolumn{3}{|c|}{$\bar{x}$ Size of PDU } & \multirow{2}{*}{ Sig. } & \multirow{2}{*}{ Scheffe's Method } \\
\hline & NS (1) & NNS-H (2) & NNS-L (3) & & \\
\hline Title (6 w) & 5.14 & 5.60 & 5.80 & .396 & - \\
\hline S1 (26 w) & 9.91 & 5.91 & 4.80 & $.000^{*}$ & $(1)>(2),(1)>(3)$ \\
\hline $\mathrm{S} 2(22 \mathrm{w})$ & 7.07 & 6.33 & 5.08 & .050 & - \\
\hline S3 (33 w) & 6.80 & 5.03 & 3.95 & $.000^{*}$ & $(1)>(2),(1)>(3)$ \\
\hline S4 (16 w) & 7.24 & 4.66 & 4.62 & $.007^{*}$ & (1) $>$ (2), (1) > (3) \\
\hline S5(16 w) & 6.28 & 5.28 & 4.44 & $.032^{\star}$ & $(1)>(3)$ \\
\hline
\end{tabular}

$* \bar{p}<.05$

As displayed in Table 5, it can be observed that the average numbers of PDU in all 5 sentences, except the title statement, were linear across the groups. The number was lowest in the NS group and highest in the low group. It should be noted that the title of a text, which typically contains a small number of words, is normally intended to capture the attention of the hearer. The use of pitch and pause when reading the title may vary depending on inter-speaker variability in rhetoric and stylistic preferences. In this study, however, most participants did not pause within the 6 -word title. The result showed that only a few participants divided the title into 2 chunks. With regard to the number of PDU in the 5 sentences, one may observe that the performance between the NS group and the low group differed significantly in all 5 sentences. Between the NS group and the high group, the difference was not statistically significant. The results suggest that the number of pause produced by the high group appeared to be more native-like than that of the low group.

With respect to PDU size as demonstrated in Table 6, it was found that the average ratio of word per PDU linearly increased in relation to proficiency levels. Again, except for the title statement, the NS group processed information in bigger and longer chunks among all groups. Within the 2 Thai learner groups, participants in the high group read in longer units than those in the low group. A notable point observed from Table 6 is the average PDU in sentence 3 . The results show that the low group divided this 33-word sentence into 3.95 words per pause, which appears to be the shortest chunk size among all the 5 sentences. This might suggest that the lower proficiency level learners had more difficulty processing information in the sentence that is relatively longer and more complex than the remaining 4 sentences. (Refer to the data on pause locations of the low group in Section 3.4)

In terms of PDU size, statistical testing results revealed significant difference between the NS group and the low group in sentences 1, 3, 4 and 5. Between the NS group and the high group, significant difference was found in sentences 1, 3 and 4. Between the 2 groups of Thai learners, difference in PDU size shows no statistical significance.

\subsection{Pause Location}

As mentioned earlier, the placement of pause has been found to relate mainly to the syntactic structures of sentences. Thus, in examining pause location, it is necessary to predict the positions in which potential pause sites are likely to occur in the reading text. Accordingly, the text was initially analyzed and marked with the double slash (I/) and single slash (I) at strong and weak boundaries as described in Section 2.2 above. The predicted pause sites were then used as the framework to analyze the participants' pause positions in order to identify the extent to which the high and low groups used grammatically appropriate pausing.

The data set below shows pause locations exhibited by each group of participants. The numbers in parentheses indicate the number of participants producing pauses and the single slash $(I)$ and double slash $(I /)$ indicate pauses predicted on the basis of syntactic structures. In the data set, there are 14 locations (i.e. 6 at sentence finals and 8 at major clause boundaries) predetermined to be strong boundaries and are marked with (II). Eighteen other locations, marked with (/) for weak boundaries between phrases, are potential but optional pause sites. 


\subsubsection{NS Group (n=7)}

[Title] The North Wind (2)/ and The Sun (7)//

[S1] One day, (3)/ the North Wind and the Sun / were arguing / about which of them was stronger, (7)// when a traveler came along (2)/ wrapped up in an overcoat.(7)// [S2] They agreed (7)// that the one / who could make the traveler (1) take his coat off (7)// would be considered / stronger / than the other one. (7)//

[S3] Then (1)/ the North Wind (1)/ blew as hard as he could, (7)// but the harder he blew, (7)// the tighter (1)/ the traveler wrapped his coat around him; (7)// and at last (4)/ the North Wind (1)/gave up trying. (7)//

[S4] Then (1)/ the Sun began to shine hot, (7)// and right away / the traveler (1) took his coat off. (7)// [S5] And so (3)/ the North Wind (2)/ had to admit (7)// that the Sun was stronger / than he was. (7)//

\subsubsection{NNS-H Group (n=15)}

[Title] The North Wind (2)/ and The Sun (15)//

[S1] One day, (13)/ the North Wind (1) and the Sun (5)/ were arguing / about (8) which of them (2) was stronger, (15)// when a traveler came along (8)/ wrapped up (5) in an overcoat. (15)// [S2] They agreed // that (14) the one (1)/ who could make the traveler (2) take his coat off (15)// would be considered (6)/ stronger (2)/ than the other one. (15)//

[S3] Then (12)/ the North Wind / blew (1) as hard as he could, (15)// but (5) the harder he blew, (15)// the tighter (3)// the traveler (1) wrapped (1) his coat (2) around him; (15)// and at last (15)/ the North Wind (1)/ gave up trying. (15)//

[S4] Then (10)/ the Sun began (1) to shine hot, (15)// and (1) right away (10)/ the traveler (3) took his coat off. (15)// [S5] And so

(10)/ the North Wind (1)/ had to admit (2)// that (13) the Sun (2) was stronger (4)/ than he was. (15)//

\subsubsection{NNS-L Group (n=15)}

[Title] The North Wind (1)/ and The Sun (15)/l

[S1] One day, (10)/ the North Wind (1) and the Sun (6)/ were (2) arguing (2)/ about (2) which (5) of (2) them (6) was (3) stronger, (15)// when (3) a traveler (5) came along / wrapped (7) up (9) in (2) an (1) overcoat. (15)// [S2] They (2) agreed (5)// that (5) the one (7)/ who (1) could (5) make (1) the (1) traveler (6) take (2) his (2) coat (3) off (8)// would (1) be (1) considered (9)/ stronger (2)/ than (3) the other one. (15)//

[S3] Then (2)/ the North (1) Wind / blew (6) as (3) hard (9) as (4) he (2) could, (15)// but (7) the harder (4) he blew, (15)// the (2) tighter (7)/ the (2) traveler (5) wrapped (10) his (3) coat (1) around him; (15)// and (2) at last (6)/ the North (2) Wind (4)/ gave (1) up trying. (15)/l

[S4] Then (2)/ the Sun (4) began (3) to shine hot, (15)// and (1) right (1) away (5)/ the (1) traveler (6) took (4) his (5) coat (2) off. (15)// [S5] And (2) so (3)/ the North (2) Wind (5)/ had (6) to admit (9)// that (7) the Sun (2) was (1) stronger (7)/ than (2) he (1) was. (15)/l

From the data set above, one may observe that all participants paused at sentence boundaries without exception. Variability existed in the occurrence of pauses within sentences. Among the NS controls, a uniform pattern of pause placement existed at all 14 boundaries judged as strong. Almost all cases of inconsistency occurred at weak boundaries between phrases. It is notable that 1 native reader paused at a place other than those predetermined to be boundaries between sense groups, i.e. at the subject-predicate (S-P) juncture: the traveler + take in sentence 2 and the traveler + took in sentence 4.

Within the high group, uniformity existed at 13 pause locations, 12 of which were in agreement with predetermined pauses at strong boundaries. It is worth noting that at 2 clause boundaries, i.e. junctures between the verb and the 'thatnoun clause' (i.e. they agree + that in sentence 2 and admit + that in sentence 5), most NNS-H participants performed a pause pattern differently from that of native speakers. This verb-noun clause juncture is predicted to be grammatically appropriate for a pause because it is a point where a main clause and a subordinate clause join together. A pause should be placed before 'that' because the complementizer 'that' is considered an integral part of the subordinate clause in English and it should not be interrupted within the clause. One may observe that in these 2 environments, most participants in the high group made a pause after 'that' in both sentences; only 2 participants placed a pause before 'that' in sentence 5. This phenomenon could presumably be hypothesized to arise from the transfer of a similar element or pattern in the learners' native language. The word 'that' can be translated into 'waa' in Thai. However, 'waa' does not function as a complementizer as 'that' does in English. In Thai grammar, 'waa' is claimed to function as a particle attached to a verb in a Thai serial verb construction and can not be separated from the verb by a pause. Therefore, when saying a sentence with the-'waa' noun clause, Thai speakers normally make a pause after the word 'waa'. Likewise, in 
the Thai writing system in which words are normally written continuously without space except at the sentence and clause boundaries, the particle 'waa' has to be attached to the verb; and a space is required to be placed after it. From the results of this study, the majority of participants in the high group paused after 'that' in both sentences. It could thus be hypothesized that such a pattern is likely to be influenced by the transfer of learners' L1 to L2 English.

In the low group, a uniform pattern of pause placement was found at 11 locations: 6 at sentence boundaries and 5 at major clause boundaries. A large number of pauses occurred at locations which were syntactically less likely pause sites, many of which appeared to violate phrase structure rules. Those environments included:

1. Within a verb phrase

2. Between an adjective and the noun it modified

3. Between a noun and its determiner

4. Between a preposition and its object

5. Between a verb and its direct object

6. Between a copula verb and a complement

7. Between a simple subject and a verb

8. Before a prepositional phrase

9. After the conjunction

Another notable point was observed in the use of pause in the low group at the 'that-noun clause' juncture. The data indicate a different pattern from that of the high group. In the low group, pauses occurred both preceding and following 'that' in both sentences. This suggests that the theory of L1 transfer may not be used to explicate the pattern of learners with lower English proficiency. One possible explanation for this phenomenon could be attributed to the learners' limited syntactic knowledge, which may have caused them to be unaware of the syntactic similarities and differences between the two languages. As supported by the data, learners in the low group made numerous grammatically inappropriate pauses.

Comparatively, the findings indicate that participants with lower English proficiency paused substantially more frequently, and thus produced shorter size of PDU, than the higher-proficiency learners. In addition, the pause patterns of the low group were more varied; many pauses were found at locations which did not correlate with English syntactic units. This suggests that with insufficient knowledge of the syntactic and semantic ties between words and phrases, learners will face with difficulty using appropriate phrasing and pausing.

\section{Summary and Discussion}

The experiment using a reading-aloud task was conducted in order to investigate the pause patterns in read speech of Thai EFL learners in contrast with those of native English speakers. Additionally the experiment served to examine the extent to which the pause patterns of native English speakers and Thai learners at high and low English proficiency levels exhibit relations to syntactic structures of English sentences.

Considering the number of pauses and PDU size, the results reveal that learners with lower English proficiency read the 119-word text in more number of chunks than the higher proficiency learners, and consequently, their chunks of information (PDU) contained a smaller number of words. Native speakers, on the other hand, divided the text into longer PDUs and therefore paused less than the 2 groups of Thai learners. With respect to the number of pauses, statistical testing results showed that the performance between the NS and the high group did not differ significantly. However, in terms of PDU size, statistical testing showed that the performance of the NS group differed significantly from the performance of both the high and low groups. This probably suggests that the ability to process language in proper length of chunks could be claimed to be achieved at a more advanced level of language proficiency.

In examining pause patterns at the sentence level, the findings show that, between the NS group and the low group, the difference in number of pauses was significant in all 5 sentences except for the title statement, but the difference in performance between the NS and the high groups showed no statistical significance. With respect to PDU size, significant difference between the NS and the low groups existed in 4 sentences; whereas between the NS and the high groups, significant difference was found in 3 sentences.

In the investigation of pause locations, it was found that all NS participants paused uniformly at the 14 positions that had been predetermined to be strong boundary pause sites. Almost all cases of inconsistency occurred at weak boundaries between phrases. The results of pause position among the native readers support earlier studies which stated that pause positions mainly correlate with syntactic structures, particularly at major syntactic edges. Among the learners in the high group, the findings indicate that most pauses occurred between major syntactic boundaries-such as 
sentences and clauses-and were in agreement with predicted pause sites. Additional pauses were made between phrases at predicted weaker boundaries. Pauses made at grammatically inappropriate pause locations were substantially fewer in number than those made by the low group. As can be seen, learners in the low group made grammatically appropriate pauses mainly at sentence boundaries and major clause boundaries, which were signaled by punctuation. Several other pauses found in the data of the low group occurred between words or within the syntactically related groups of words that should not be interrupted by a pause. Thus, it can be summarized that learners with higher English proficiency seem to have more syntactic related pauses between sentences, clauses and phrases, while lower proficiency learners tend to make numerous pauses at word boundaries probably because they had limited knowledge with regard to the relationships between structural parts of sentences.

One point that should be noted is the learners' placement of pause in the 'that-noun clause' structure. The results reveal that learners in the high group paused differently from the native speaker norm. Instead of pausing in front of the noun clause complementizer 'that' in the same way native speakers did, most participants paused between 'that' and the clause that follows it. This phenomenon is hypothesized to result from the transfer of learners' L1 to L2 English. However, learners in the low group paused differently. Their pauses occurred both preceding and following 'that'. It could be assumed that the pattern in the low group could result from their limited knowledge of syntactic structures as discussed earlier.

In summary, with respect to the first objective of the study, the results indicate that learners with higher English proficiency produced pause patterns that were more native-like than lower proficiency learners, and that processing information in proper chunk size was quite problematic. In relation to the second objective, it was found that learners in the low group produced less syntactic related pauses than those in the high group, suggesting that acquisition of syntactic knowledge may be achieved at a later stage of second language development. With increasing proficiency, learners tended to become more aware of syntactic and semantic ties between words and phrases. As a result, their pause patterns exhibited more similarities to the NS norm in terms of relations to syntactic structures of sentences.

\section{Implications}

This study addressed the problems in using appropriate pausing among Thai learners in EFL contexts. The findings of this study offer some pedagogical implications. First, teachers should attempt to show their students that pause plays an essential role in oral communication. Second, the teacher's goal should also be to help students place pauses properly. It is hoped that the findings will shed some lights into the teaching of proper models of pause by integrating reading aloud as part of the EFL teaching/learning processes.

\section{Recommendations}

Based on the main findings of the study, the following recommendations are made for further research.

Firstly, the participants in this study were not asked to state the reason why they paused at particular locations. Therefore, it limits one's ability to explicate the patterns of their performance or provide a thorough account of what processes are involved when the participants pause. For a more in-depth analysis of the processes involved in phrasing and pausing, further research is recommended that investigates the reason why participants pause at each location whether by think-aloud protocols or retrospective interviews.

Secondly, the analyses in this study have provided an overview of pause patterns of 2 levels of Thai learners. Continued research can be conducted to qualitatively examine the characteristics of information chunks produced by Thai learners at varying developmental stages in greater detail.

\section{References}

Bada, E. (2006). Pausing preceding and following 'that' in English. ELT Journal, 60, 125-132.

Bada, E. \& Genc, B. (2008). Pausing preceding and following to in to-infinitives: A study with implications to reading and speaking skills in ELT. Journal of Pragmatics, 40, 1939-1949.

Grosjean, F., Grosjean, L. \& Lane, H. (1979). The patterns of silence: Performance structures in sentence production. Cognitive Psychology, 11, 58-81.

Keating, P. \& Shattuck-Hufnagel, S. (2002). A prosodic view of word form encoding for speech production. UCLA Working Papers in Phonetics, 101, 112-156.

Krivokapi, J. (2007). Prosodic planning: Effects of phrasal length and complexity on pause duration. Journal of Phonetics, 35, 162-179. 
Luksaneeyanawin, S. (1988). A research report on characteristics and functions of pause in Thai. Linguistic Research Unit, Faculty of Arts, Chulalongkorn University. (In Thai)

Riazantseva, A. (2001). Second language proficiency and pausing: A study of Russian speakers of English. Studies in Second Language Acquisition. 23, 497-526.

Selkirk, E.O. (1984). Phonology and Syntax: The Relations Between Sound and Structure. Cambridge, Massachusetts: MIT Press.

Taylor, D.S. (1981). Non-native speakers and the rhythm of English. In A. Brown (Ed.), Teaching English pronunciation (pp. 235-244). London: Routledge.

Truckenbrodt, H. (1999). On the relation between syntactic phrases and phonological phrases. Linguistic Inquiry, 30, 219-255.

Wennerstrom, A. (1994). Intonational meaning in English discourse: A study of non- nativespeakers. Applied Linguistics, 15, 399-420.

Zellner, Brigitte. (1994). Pauses and the temporal structure of speech, in E. Keller (Ed.)

Fundamental of speech synthesis and speech recognition. (pp. 41-62). Chichester: John Wiley.

Zvonik, E. (2004). Pausing and temporal organization of phrases: An experimental study of read speech. Ph.D. Dissertation, Department of Computer Science, University College Dublin, National University of Ireland. 